\title{
“No Anastomosis” Combined Colon Conduit and Colostomy Diversion with Pelvic Exenteration: An Underutilized, Cost-Effective Technique Reducing Bowel Complications
}

\author{
Sayyid KR, Neal DE, Albo D, Kruse EJ, Wallbillich JJ, Rungruang BJ, Ghamande SJ and Martha K Terris* \\ Department of Urology, Augusta University, USA \\ *Corresponding author: Martha K Terris, Department of Urology, Medical College of Georgia, Augusta University, Augusta, GA, USA
}

Submission: 眥January 31, 2018; Published: 眥February 12, 2018

\section{Opinioin}

Since its initial description by Brunschwig in 1948 [1], pelvic exenteration has become an established procedure to treat locally advanced or recurrent gastrointestinal, gynecologic and urologic malignancies as well as after failure of other options for radiation cystitis/colitis and neurogenic bladder/bowel. This procedure typically involves en bloc removal of the remaining pelvic viscera: bladder, rectum, and prostate/seminal vesicles in males or uterus and vagina (partial or complete) in females [2]. Given the extensive resection and reconstruction involved, frequent prior radiation, and prevalence of adhesions from multiple prior procedures, perioperative complications are commonly encountered $[3,4]$. Such complications may prolong the hospital length of stay, and consequently increase overall healthcare costs. Anastomotic leak, post-operative ileus, and bowel obstruction have been shown in previous studies to be the most important factors contributing to a prolonged hospital stay following a pelvic exenteration [5]. Anastomotic leaks have been shown, on average, to prolong hospital length of stay between 19 and 36 days with many patients requiring pelvic drain placement and intravenous antibiotic use. Thus, urinary and fecal diversion surgical techniques that avoid bowel anastomosis have the potential to decrease peri- and postoperative morbidities with resultant decreased hospital length of stay and lower health care costs $[5,6]$.

Davis and Noble described in 1992 a surgical technique that involves the use of a pre-existing colostomy as an intestinal conduit for urinary diversion in patients with neurogenic bladder/bowel, radiation cystitis/colitis, and metachronous colon and bladder cancer. This technique involves the isolation of a 20 to $30 \mathrm{~cm}$ segment of colon proximal to the initial colostomy that is divided and transformed into a colon conduit by anastomosing the ureters to the segment. The proximal bowel is subsequently fashioned into a new colostomy [7]. The main advantage of this technique is that it eliminates the need for a bowel anastomosis, which is required with ileal conduits [6]. It also avoids the mixing of fecal and urine output that is characteristic of wet colostomies, which may be associated with higher rates of ascending pyelonephritis [7]. Electrolyte disturbances do not seem to be significantly different when comparing colon and ileal conduits [8]. Furthermore, use of transverse colon with this technique is also convenient, as it permits use of non-irradiated bowel in patients who have previously received pelvic radiation [9].

In our experience, using the most distal available colon as a urinary colon conduit and the more proximal colon as a colostomy (Figure 1), avoidance of an entero-enteric anastomosis improves recovery time, decreases complications, and improves length of stay. We favor a Wallace anastomosis to decrease the risk of stricture in potentially irradiated distal ureters. We find the ureteral anastomosis is made easier in these cases as there is no necessity to pass one of the ureters under the colonic mesentery [10].

We would also like to emphasize the importance of a multidisciplinary approach to such surgeries. Given the multiorgan nature of pelvic exenteration, utilizing the different, the complimentary expertise and technical skills of multiple surgical services may lead to superior outcomes. A study assessing predictors of length of hospital stay following a pelvic exenteration demonstrated that gastrointestinal surgical team involvement decreased hospital length of stay by five days. Surprisingly, despite $99 \%$ of patients having gastrointestinal tract surgical manipulation, only $60 \%$ of cases had gastrointestinal surgical team involvement. These numbers highlight the importance of increased communication and collaboration between different surgical services in order to optimize patient outcomes [5].

In summary, we believe variations of that the relatively simple surgical technique described by Davis and Noble, offer the unique possibility of combined urinary and fecal diversion with no required bowel anastomosis in patients undergoing pelvic 
exenteration., potentially decreasing peri-operative complications and its associated sequelae [7]. A multi-disciplinary approach that utilizes the technical skills and expertise of multiple surgical services is crucial towards optimizing patient outcomes.
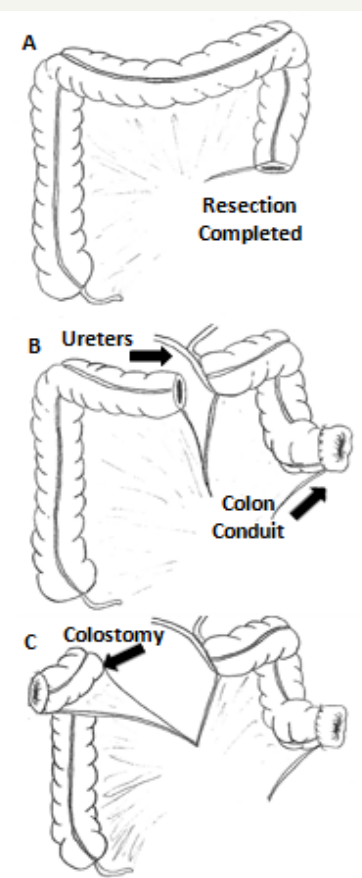

Figure 1: "No Anastomosis" Combined Colon Conduit and Colostomy Diversion.

A. After successful removal of bladder, rectum, and any necessary gender-specific organs,

B. The most distal available colon is utilized to create a urinary colon conduit with Wallace ureteral anastomosis, and

C. The more proximal colon, typically the transverse colon, is brought to the other side as a colostomy.

\section{References}

1. Brunschwig A (1948) Complete excision of pelvic viscera for advanced carcinoma; a one-stage abdominoperineal operation with end colostomy and bilateral ureteral implantation into the colon above the colostomy. Cancer 1(2): 177-183.

2. Heger U, Koch M, Buchler MW, Weitz J (2010) Total pelvic exenteration. Chirug 10(81): 897-901.

3. Pawlik TM, Skibber JM, Rodriguez-Bigas MA (2006) Pelvic exenteration for advanced pelvic malignancies. Ann Surg Oncol 13(5): 612-623.

4. Rodriguez-Bigas MA, Petrelli NJ (1996) Pelvic exenteration and its modifications. Am J Surg 171(2): 293-298.

5. Guo Y, Chang E, Bozkurt M, Park M, Liu D, et al. (2017) Factors affecting hospital length of stay following pelvic exenteration surgery. J Surg Onc pp. 24878.

6. Teixeira SC, Ferenschild FT, Solomon MJ, Rodwell L, Harrison JD, et al (2012) Urological leaks after pelvic exenterations comparing formation of colonic and ileal conduits. Eur J Surg Oncol 38(4): 361-366.

7. Davis BE, Noble MJ (1992) Simplified urinary diversion in patients with pre existing or imminent colostomy. J Urol 147(5): 1245-1247.

8. Lopes de Queiroz F, Barbosa-Silva T, Pyramo Costa LM, Werneck Côrtes BJ, Figueiredo JA, et al. (2006) Double-barreled wet colostomy with simultaneous urinary and faecal diversion: results in 9 patients and review of the literature. Colorectal Dis 8(4): 353-359.

9. Gan J, Hamid R (2017) Literature Review: Double-Barreled Wet Colostomy (one Stoma) versus Ileal Conduit with Colostomy (Two Stomas). Urol Int 98(3): 249-254.

10. Kouba E, Sands M, Lentz A, Wallen E, Pruthi RS (2007) A comparison of the Bricker versus Wallace ureteroileal anastomosis in patients undergoing urinary diversion for bladder cancer. J Urol 178(3 Pt 1): 945-948.

\section{Your subsequent submission with Crimson Publishers will attain the below benefits}

International License

For possible submission use the below is the URL Submit Article
High-level peer review and editorial services

- Freely accessible online immediately upon publication

- Authors retain the copyright to their work

- Licensing it under a Creative Commons license

- Visibility through different online platforms

- Global attainment for your research

- Article availability in different formats (Pdf, E-pub, Full Text)

- Endless customer service

- Reasonable Membership services

- Reprints availability upon request

- One step article tracking system 